\title{
Implementation of OFDM Transceiver using GMSK and QPSK Encoding Technique
}

\author{
Gunjan Negi \\ Student, ECE \\ GRD Institute of Management \\ and Technology \\ Dehradun, India
}

\author{
Arun Kumar \\ Assistant Professor, ECE \\ GRD Institute of Management \\ and Technology \\ Dehradun, India
}

\author{
Anuj Saxena \\ Information Technology \\ Karnataka State University \\ Karnataka, India
}

\begin{abstract}
OFDM (Orthogonal Frequency Division Multiplexing) transmissions are arising as important modulation techniques because of its robustness against interference. Various modulation schemes have been used to implement OFDM. In this paper, the OFDM transceiver system is implemented using MATLAB. Gaussian Minimum Shift Keying modulation technique has been implemented in the proposed OFDM. The bit error rate (BER) performance has been evaluated in AWGN (Additive White Gaussian Noise) channel and is compared to Quadrature Phase Shift Keying modulation scheme.
\end{abstract}

\section{General Terms}

Bit error rate, Matlab, Additive White Gaussian Noise.

\section{Keywords}

Orthogonal Frequency Division Multiplexing, Gaussian Minimum Shift Keying, Quadrature Phase Shift Keying

\section{INTRODUCTION}

OFDM can be implemented by using various modulation techniques. Researchers have proposed various modulation techniques like QPSK, QAM, and MSK etc. This paper demonstrates the simulation of an OFDM transceiver using Gaussian Minimum Shift Keying modulation technique and Quadrature Phase Shift Keying technique.

The paper is organized as follows. Section II gives the implementation of the OFDM transceiver with OFDM system requirements and specifications. Section III gives the experimental results of system evaluation in term of simulation environment. Section IV includes conclusions of OFDM implementation.

\subsection{System Design}

The general structure of OFDM transceiver system using Matlab simulation is illustrated in figure 1 .

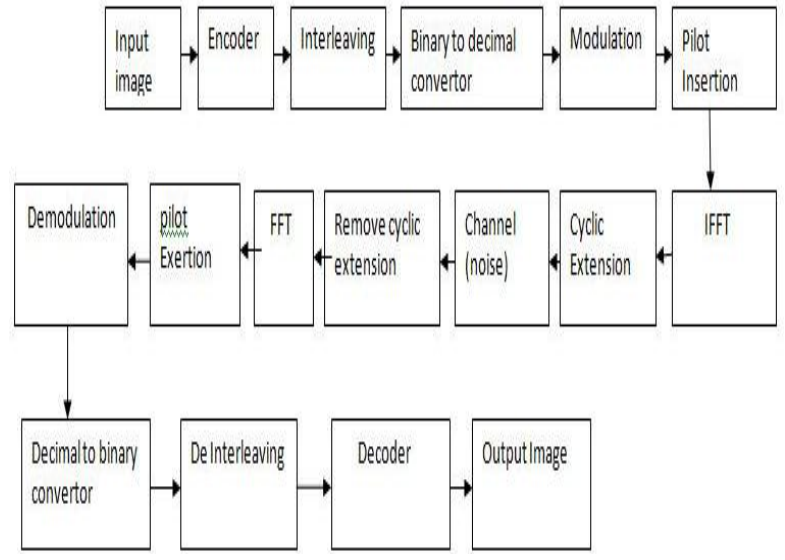

Figure 1: Block Diagram of OFDM Transceiver

\subsubsection{Transmitter}

In an OFDM system, the data block of $\mathrm{N}$ symbols, denoted by

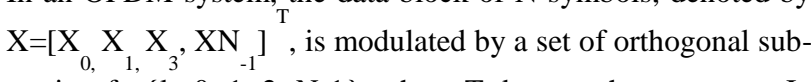
carrier, $\mathrm{f}_{\mathrm{k}},\{\mathrm{k}=0,1,2, \mathrm{~N}-1\}$, where $\mathrm{T}$ denotes the transpose. In OFDM system to sustain the orthogonality of the signals, the spacing $\Delta \mathrm{f}$ between neighboring subcarriers is set to be a multiple of $1 / \mathrm{T}$. i.e., $\Delta \mathrm{f}=\mathrm{m} 1 / \mathrm{T}$, where $\mathrm{T}$ is the duration of an OFDM symbol, then the transmitted OFDM symbol is given by

$\mathrm{x}(\mathrm{t})=1 / \mathrm{N} \sum_{k=0}^{N-1} \mathrm{X}(\mathrm{k}) \mathrm{e}^{\mathrm{j} 2 \pi \mathrm{ft}} \quad 0<=\mathrm{t}>=\mathrm{T}$

The Input image is converted to source data and is passed through the encoder. Convolution codes are used to encode the data. Then the signal is passed through interleaver.Interleaving increases the resistance to fading. Binary to decimal convertor converts the binary vector to decimal number.

\subsubsection{Modulator}

(a) GMSK modulator

The signal is then passed through modulator (GMSK). Gaussian Minimum Phase Shift Keying is a continuous phase modulation scheme which is generated by filtering NRZ data with a Gaussian shaping filter. The GMSK modulated waveform is characterized by the BT product. Where, B is the bandwidth of the Gaussian filter and $\mathrm{T}$ is the duration of the data bit.

The resulting signal is represented by

$S(t)=a_{I}(t) \cos (\pi t / 2 T) \cos \left(2 \pi f_{c} t\right)-a_{Q}(t) \sin \left(2 \pi f_{c} t\right)$

Where, $\mathrm{a}_{\mathrm{I}}(\mathrm{t})$ and $\mathrm{a}_{\mathrm{Q}}(\mathrm{t})$ are the even and odd information respectively. $\mathrm{a}_{\mathrm{I}}(\mathrm{t})$ has pulse edges on $\mathrm{t}=\{-\mathrm{T}, \mathrm{T}, 3 \mathrm{~T} .$.$\} and \mathrm{a}_{\mathrm{Q}}(\mathrm{t})$ on $\mathrm{t}=\{0,2 \mathrm{~T}, 4 \mathrm{~T} \ldots \ldots\}$. The carrier frequency is $\mathrm{f}_{\mathrm{c}}$.

This equation can be rewritten in a form of phase and frequency modulation,

$S(t)=\cos \left[2 \pi f_{c} t+b_{k}(t) \pi t / 2 T+Q_{k}\right]$

where $b_{k}(t)$ is +1 when $\mathrm{a}_{\mathrm{I}}(\mathrm{t})=\mathrm{a}_{\mathrm{Q}}(\mathrm{t})$ and -1 if they are of opposite

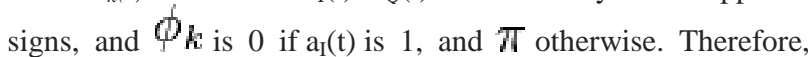
the signal is modulated in frequency and phase, and the phase changes continuously and linearly.

\section{(b)QPSK modulator}

In QPSK modulation, the input binary data is first converted to bipolar NRZ type of signal. The demultiplexer divides the signal into two separate bit streams. Here, $b_{e}(t)$ represents even numbered sequence and $b_{o}(t)$ represents odd numbered sequence. The bit stream $b_{e}(t)$ modulates carrier $\sqrt{P_{s}} \cos \left(2 \pi f_{c} t\right)$ 
and $b_{o}(t)$ modulates $\sqrt{P s}\left(\sin 2 \pi f_{c} t\right)$. The two modulated signals are:

$$
\begin{aligned}
& S_{e}(t)=b_{e}(t) \sqrt{P s}\left(\sin 2 \pi f_{c} t\right) . \\
& S_{0}(t)=b_{o}(t) \sqrt{ } P_{s} \cos \left(2 \pi f_{c} t\right)
\end{aligned}
$$

The output of the adder is the QPSK signal and is given by:

$$
\begin{aligned}
& S(t)=S_{e}(t)+S_{o}(t) \quad(6) \\
& S(t)=b_{o}(t) \sqrt{ } P_{s} \cos \left(2 \pi f_{c} t\right)+b_{e}(t) \sqrt{P s}\left(\sin 2 \pi f_{c} t\right)
\end{aligned}
$$

After modulation the signal is passed through pilot insertion. Pilots are the unmodulated data sequences which are transmitted along with the data. They are used for synchronization and channel estimation purposes. Inverse fast Fourier transform (IFFT) is basically used to transform the signals on different carriers from frequency domain to time domain.

$$
\mathrm{x}(\mathrm{n})=1 / N \sum_{k=0}^{N-1} \mathrm{X}(\mathrm{K}) \mathrm{e}^{\mathrm{j} 2 \pi \mathrm{kn} / \mathrm{N}} \mathrm{n}=0,1,2 \ldots \ldots \mathrm{N}-1
$$

Guard intervals or cyclic extension are added to the signal so as to eliminate Inter Symbol Interference.

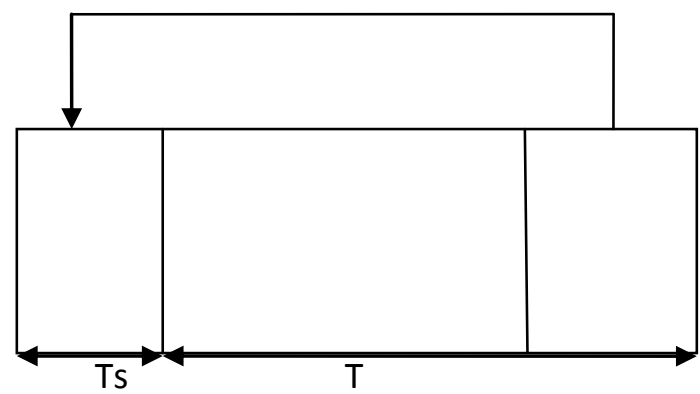

Figure 2: Cyclic Prefix

Where $\mathrm{T}$ is the length of one OFDM symbol and $s T$ is the length of $\mathrm{CP}$ in time domain respectively in Figure 2. The shadowed part in Figure 2 is the cyclic prefix. It copies the rear part of the OFDM symbol and puts it to the front of the symbol, so that the period will increase from $\mathrm{T}$ to $\mathrm{T}+\mathrm{Ts}$ and $\mathrm{s} \mathrm{T}$ is the Cyclic Prefix.

\subsubsection{Channel}

Additive White Gaussian Noise is added to the channel.

The probability density function of Gaussian noise is given by:

$\left.P_{x}(x)=1 / \sqrt{ } 2 \pi\left(e^{-(x-m}\right)^{\wedge} 2\right) / 2 \sigma^{2}$

\subsubsection{Receiver}

At the receiver, the signal is passed through the decoder.Vertibi algorithm is used as a decoding algorithm. The signal is then passed through deinterleaver. It restores the ordering of symbols. Decimal to binary convertor converts the decimal number to the binary vectors.

The signal is then passed through the QPSK and GMSK demodulator. The demodulator demodulates the OFDM signal and moves it back to the baseband signal. The fast Fourier transform is basically needed to transform signal from time domain into the frequency domain. The pilot extension block removes the pilot data sequences which are added at the transmitter.

The added cyclic extension is also removed from the received noisy signal. And we receive the output image.

\section{IMPLEMENTATION}

The steps for implementation are as follows:

Initialize required variables

Step 1. $\mathrm{fp} \leftarrow$ read image file

Step 2. [or oc on] $\leftarrow$ get size of image

Step 3. Rimage $\leftarrow$ reshape image

Step 4. t_data $\leftarrow$ convert image to logical form

Step 5. for $\mathrm{d}=0: 1: 9$

Step 6. data $\leftarrow$ divide into packets

Step 7. trellis $\leftarrow$ convolutional code polynomials to trellis

Step 8 . codedata $\leftarrow$ Convolutionally encode binary data

Step 9. End For

Step 10. $S \leftarrow$ get size

Step 11. matrix $\leftarrow$ reshape

Step 12. intlvddata $\leftarrow$ Interleave

Step 13. dec $\leftarrow$ convert to decimal

Step 14. y $\leftarrow$ modulate using GMSK/QPSK

Step 15. ifft_sig $\leftarrow$ perform inverse $\mathrm{fft}$

Step 16. Add Cyclic Prefix

Step 17. Ofdm_sig $\leftarrow$ add White Gaussian Noise

At Receiver end reverse the steps 3 through 17

\section{SIMULATION AND RESULTS}

Table 1 shows the input parameters of the ofdm system simulation.

(.jpg) file has been used as the input to test performance of the ofdm transceiver. MATLAB software has been used to implement the ofdm transceiver. There are total 5 plots available in this simulation including transmitted image, received image, transmitted OFDM signal, received OFDM signal and BER plot.

Table 1: The Input Parameters

\begin{tabular}{|l|l|}
\hline Parameter & \multicolumn{1}{|c|}{ Value } \\
\hline Source Data & $(. j p g)$ Size $600 \times 400$ \\
\hline IFFT Size & 64 \\
\hline Pilot Data & 04 \\
\hline Code & Convolution Coding \\
\hline No of Carrier & 64 \\
\hline Modulation method & GMSK,QPSK \\
\hline SNR & $0-10 \mathrm{~dB}$ \\
\hline
\end{tabular}

\subsection{GMSK Technique}

Figure 3. Shows the (.jpg) image that has been used as an input for ofdm transceiver. Figure 4.Illustrates the transmitted OFDM signal.

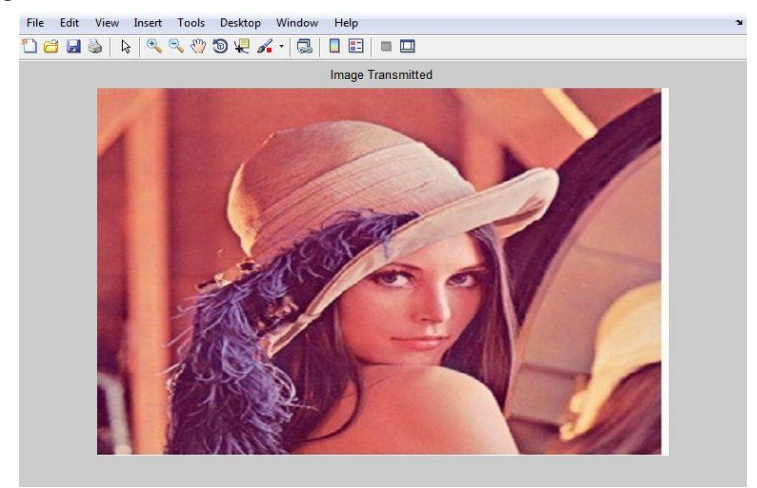

Figure 3: Transmitted input Image 


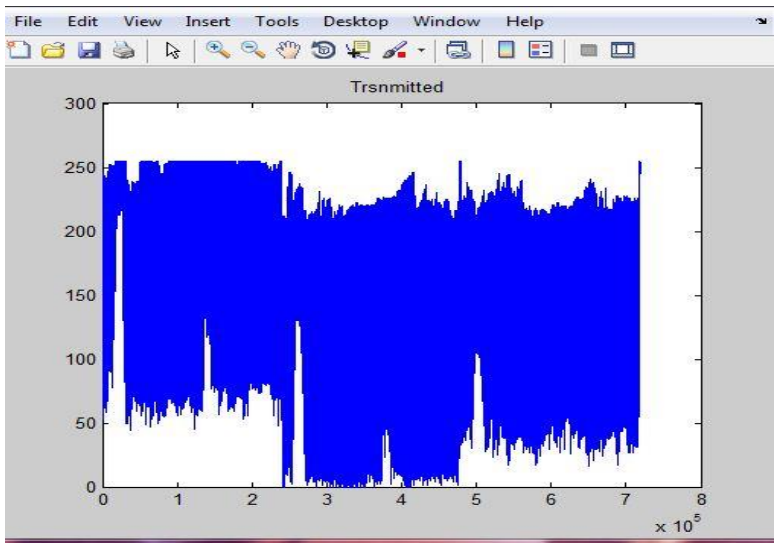

Figure 4: Transmitted OFDM signal

Figure 5 shows the received image and figure 6 shows the received OFDM signal.

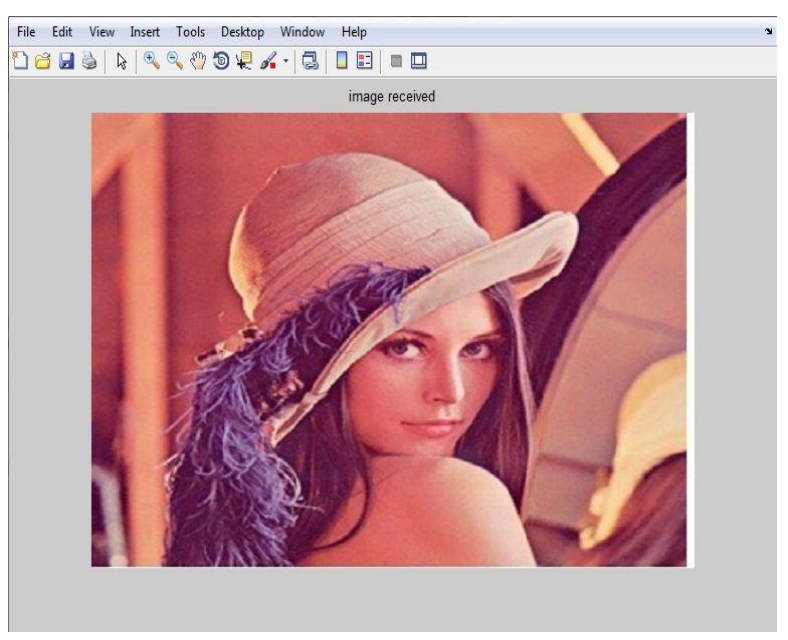

Figure 5: Received Image

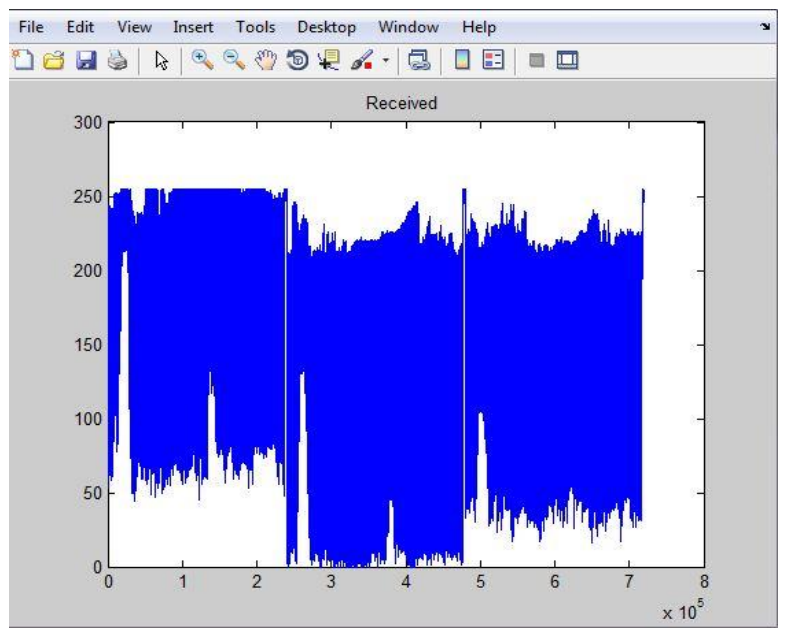

Figure 6: Received OFDM Signal

\subsection{QPSK Technique}

The same input parameters are used to implement the OFDM Transceiver using QPSK modulation technique. For the same input image, the output image and OFDM signal is obtained. Figure 7 and Figure 8 shows the received image and the received OFDM signal.

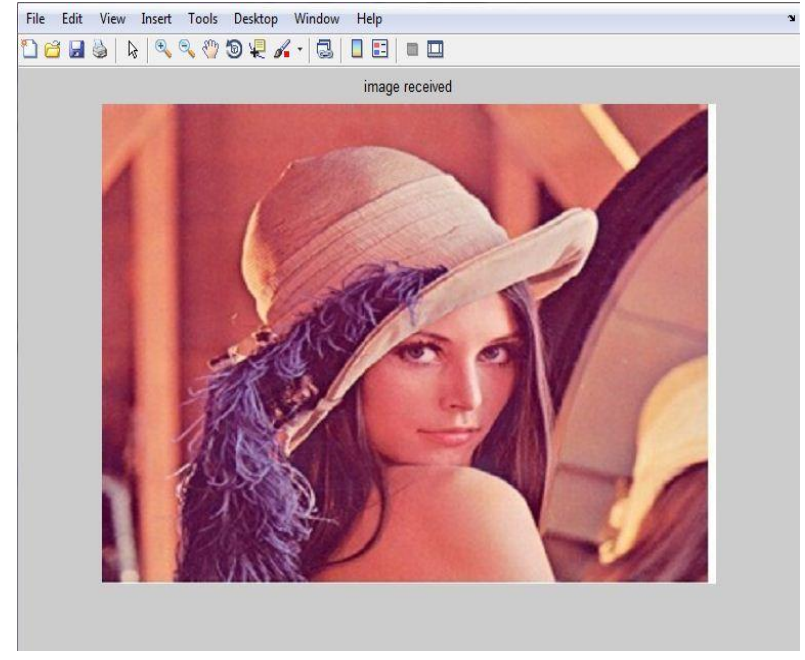

.Figure 7: Received Image

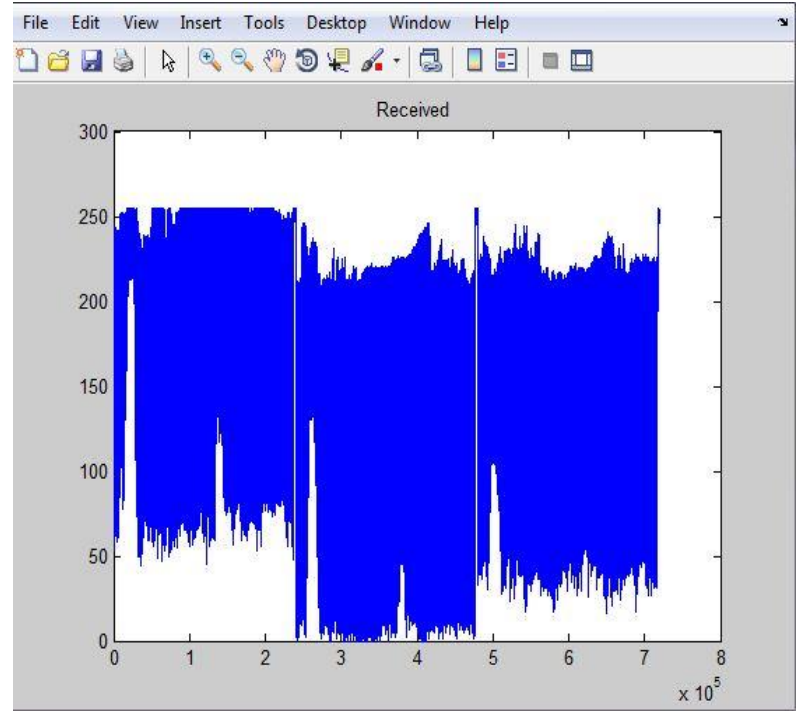

Figure 8: Received OFDM Signal

The performance of the system has been evaluated for AWGN channel, where the bit error rate analysis has been done for GMSK modulation technique and QPSK Modulation Technique. Figure 9.Shows the Bit Error Rate performance of GMSK in comparison to QPSK.

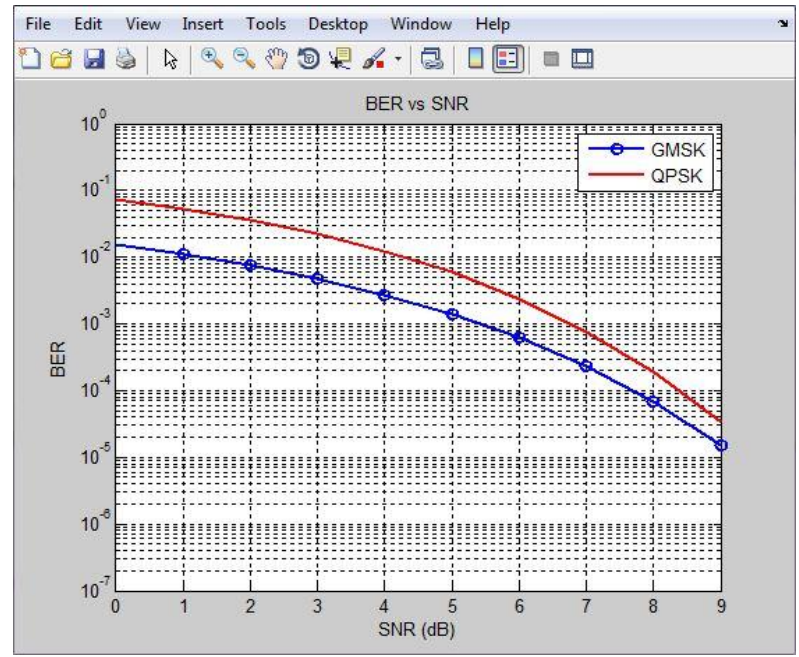

Figure 9: Simulation Results of BER Performance 


\section{CONCLUSION}

This paper describes the design of OFDM transceiver system based on simulation. GMSK and QPSK encoding technique have been used in this system to analyze the effect of modulation scheme against AWGN channel. The BER performance of GMSK has been evaluated in AWGN environment and is compared to QPSK encoding technique.

By comparing the Bit Error Rate performance of GMSK and QPSK, it is concluded that the Bit Error ate performance of GMSK is better as compared to QPSK.

The BER is comparatively high for low values of SNR (Signal to Noise Ratio). As SNR increases, the BER decreases gradually for both the encoding techniques. The observation confirms that GMSK gives best performance as compare to QPSK. The BER of GMSK can be further reduced by increasing the number of symbols. GMSK encoding technique has been used to implement OFDM transceiver. In GMSK technique, the input binary sequence is passed through a pre modulator Gaussian shaping filter. This reduces the side lobe levels of the spectrum and thus the interference between the sub carriers. But this Gaussian filter causes Inter Symbol Interference. Thus to reduce this interference, Channel Equalization algorithms could be used at the receiver end. There are various equalization techniques that can be adopted such as DFE, ZF equalization, MLSE etc.

\section{ACKNOWLEDGEMENT}

I would like to thank my guide Mr. Arun Kumar and my HOD Mr. Ankit Jha for helping me doing this research work. Without their assistance, it would not be a success.

\section{REFERENCES}

[1] Yinsheng Liu et al., "Channel Estimation for OFDM", IEEE, 2014.

[2] Dungun Kim et al., "Filter and Forward Relay Design for MIMO OFDM Systems”, IEEE July 2014.

[3] Deergha Aggarwal et al., "PAPR Reduction Using Precoding and Companding Techniques for OFDM Systems", 2015 (ICACEA)

[4] Md. Alamgir Hossain et al., "Low-Complexity Blind Phase Noise Compensation in OFDM Systems", ICEEICT 2014, IEEE.

[5] Navdeep Singh Randhawa et al., "A Survey of Equalization Techniques for an Effective Equalizer Design in MIMO-OFDM Systems", 2015, ICCPCT, IEEE.

[6] H. A. Rahim et al., "Design and Simulation of OFDM System Using DPSK Technique for Wireless LAN", ICCCE 2010

[7] H.O.Qrabil et al., "Design and Implementation of OFDM Transceiver System Using M-PSK Encoding Technique", 4th International Conference on Power Engineering, Energy and Electrical Drives Istanbul, Turkey, 13-17 May 2013.
[8] Rajesh Bansode et al., "Design, Simulation and Performance Evaluation of $4 \times 4$ MIMO Transceiver Systems Using 16 QAM”, ICWAC 2013.

[9] Behrouz Maham et al., "Impact of Transceiver I/Q Imbalance on Transmit Diversity of Beamforming OFDM Systems", IEEE, 2012.

[10] Eonpyo Hong et al., "Peak-to-Average Power Ratio Reduction for MISO OFDM Systems with Adaptive AllPass Filters", IEEE 2011.

[11] Kyung-Hwa Kim et al., "An ICI Suppression Scheme Based on the Correlative Coding for Alamouti SFBCOFDM System with Phase Noise", IEEE, 2011.

[12] Gaurav Chandra et al., "Analyzing the Effect of Modulation Order and Sub-bands on PTS and SLM PAPR Reduction Technique in Various 802.11 Standards Using Different Modulator", IJSER, 2013.

[13] Dharma Devi et al., "BER Performance of GMSK Using Matlab”, IJARCET, April 2013.

[14] S.S Ghorpade et al., "Behavior of OFDM Systems Using Matlab Simulation", IJITR, April 2013.

[15] Zain ul Abidin Jaffri et al., "Designing and Simulating the OFDM Transceiver”, IJESET, Feb 2014.

[16] S.S Ghorpade et al., "Performance Analysis of Multiband OFDM and Pulsed OFDM using Matlab Simulation", IJSR, May 2014.

[17] Enis Kocan et al., "Performance Evaluation of OFDM Amplify and Forward Relay System with Subcarrier Permutation", IEEE, 2010.

[18] Suchita Varade et al., "Performance Analysis of MIMO OFDM System Using Space Time Turbo Codes and Adaptive Beamforming”, IJCA, May 2011.

[19] T.S.Gagandeep et al., "Performance Analysis of Extended SNR Estimation for OFDM System under AWGN and Rayleigh Channel”, IJAEST, 2011.

[20] Ahasanun Nessa et al., "Performance Analysis of Two Hop Cooperative MIMO Transmission with Best Relay Selection in Rayleigh Fading Channel”, IAJIT, 2011.

[21] Mohamad Aoude et al., "Performance Analysis of QPSK OFDM with Fading, Frequency Offset and Channel Estimation Error", Journal of Theoretical and Applied Information Technology, 2010.

[22] Srabani Mohapatra et al., "Performance Enhancement of OFDM System with ICI Reduction Technique", WCE 2009.

[23] D.K Sharma et al., "Studies on Performance of Pulse Shaped OFDM Signal”, IJCSCT, Jan. 2011.

[24] V.Jagan naveen et al., "Performance Analysis of MCCDMA AND OFDM in Wireless Rayleigh Channel", IJAST, 2010.

[25] Neenu Joseph et al., "FPGA based Realization of OFDM Transceiver system for Software Defined Radio", ICDCS, 2014. 